\title{
Inflammatory Polyps: An Unreported Side Effect of Argon Plasma Coagulation
}

For about 5 years the endoscopic use of argon plasma coagulation (APC) has been spreading in gastroenterologic endoscopy. Only a few disadvantages or side effects are known, such as intestinal distension and gastrointestinal wall emphysema caused by the argon flow, or free perforation with symptomatic or asymptomatic accumulation of gas in the mediastinum or peritoneal cavity $(1,2)$.

We report a hitherto undescribed side effect of APC, noticed in a 70-year-old woman with liver cirrhosis and known iron deficiency anemia (lowest hemoglobin level $4.7 \mathrm{~g} \%$ ) of at least 18 months' duration. The cause was a GAVE syndrome, which was treated by APC.

After six treatment sessions within 3 weeks in 1996 with argon plasma coagulation of the pyloric antrum we stopped therapy, because the induced ulcers healed very slowly. Although the treatment was incom-

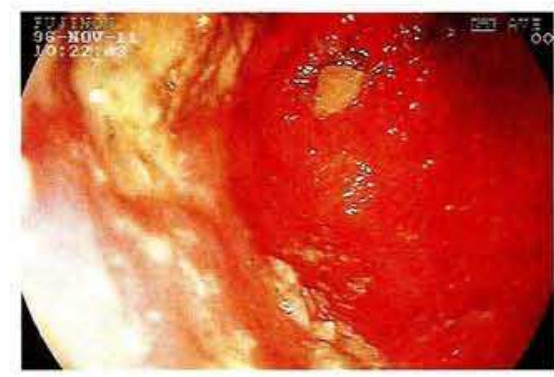

Figure 1: Ulcer on the anterior antral wall following argon plasma coagulation (APC). Note the diffuse vasectasias. plete the patient has remained stable to the present day.

Further endoscopies 6 months and 8 months after ending the coagulation treatment showed large soft polyp-like lesions at the former site of the coagulation ulcers. The polyps did not bleed spontaneously, but did so after gentle touching with the biopsy forceps.

Histologically the exophytic lesions showed an inflammatory organoid pattern. Under an eroded surface, numerous capillaries were situated in an fibromyxoid matrix, with secondary cellular inflammatory changes. Occasionally regenerating foveolar tubules were found in the marginal areas.

The patient herself had no complaints.

To our knowledge this is the first description of granulomatous inflammatory polyps as a side effect of APC therapy.

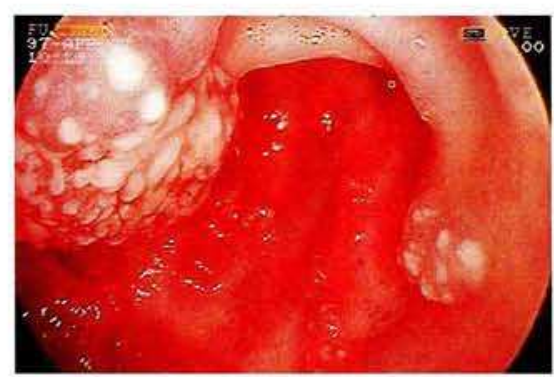

Figure 2: One large and several smaller polyplike lesions at the former sites of post-APC ulcers; 6 months later. 
H. J. Schmeck-Lindenau', W. Kurtz', M. Heine ${ }^{2}$

'Med. Dept. I, Zentralkrankenhaus

Reinkenheide, Bremerhaven, Germany

${ }^{2}$ Pathologic Institute,

Bremerhaven, Germany

\section{References}

1. Grund KE, Zindel C, Farin G. Argon plasma coagulation through a flexible endoscope. Evaluation of a new therapeutic method after 1606 uses. Dtsch Med Wochenschr 1997; 122: 432-8.

2. Tan ACITL, Schellekens PPA, Wahab P, Mulder CJJ. Pneumatosis intestinalis, retroperitonealis, and thoracalis after argon plasma coagulation. Endoscopy 1995; $27: 698-9$.
Corresponding Author H. J. Schmeck-Lindenau, M.D. Med. Dept. I

Zentralkrankenhaus Reinkenheide 27574 Bremerhaven

Germany

Fax: + 49-471-2993223 